\title{
Evaluation of the retinal layers and microvasculature in patients with acromegaly: a case-control OCT angiography study
}

\author{
Fahrettin Akay $\mathbb{1}^{1} \cdot$ Berkay Akmaz ${ }^{1} \cdot$ Mehmed Uğur Işik $\mathbb{D}^{2} \cdot$ Yusuf Ziya Güven ${ }^{1} \cdot$ Güzide Gonca Örük ${ }^{3}$
}

Received: 9 December 2019 / Revised: 31 March 2020 / Accepted: 8 April 2020 / Published online: 28 April 2020

(c) The Author(s), under exclusive licence to The Royal College of Ophthalmologists 2020

\begin{abstract}
Background The aim of study was to evaluate the retinal layers and macular capillary network with OCTA in acromegaly patients, to compare with healthy population.

Methods In this prospective, observational, and comparative study, 40 acromegaly patients and 40 healthy control participants were included. Serum IGF-1 levels and disease duration of all patients were noted. Macular layers and angiography scanning was performed with a Zeiss Cirrus 5000 OCTA system. Macular thickness, RNFL, and GC-IPL values were obtained. For central vessel and perfusion density, central $6 \mathrm{~mm}$ was obtained and was evaluated by dividing into three groups (inner, outer, full). FAZ parameters were evaluated dividing into three groups (area, perimeter, circularity index). Analysis of the data was performed with the SPSS for Windows.

Results There was no significant difference between the patient group and the control group in terms of age, gender, best corrected visual acuity (BCVA), spherical equivalent (SE), intraocular pressure (IOP), and axial length (AL). The mean follow-up period after diagnosis was $11.0 \pm 5.5$ years. Central and mean macular thicknesses were also significantly higher in the acromegaly group $(p<0.05)$. Superior, inferior, and average RNFL thicknesses were also significantly thinner in the acromegaly group $(p<0.05)$. When OCTA parameters were compared between groups, there was a significant decrease in central vessel density (CVD) and central perfusion density (CPD) values in all regions in acromegaly group compared with controls $(p<0.05)$.

Conclusion Our findings with OCTA show that acromegaly causes a significant capillary network decrease according to the healthy subjects.
\end{abstract}

\section{Introduction}

Acromegaly is a rare chronic disease caused by pituitary adenoma characterized by hypersecretion of growth hormone $(\mathrm{GH})$ and insulin-like growth factor-1 (IGF-1) [1]. Excessive secretion of GH and IGF-1 can cause skeletal growth, soft tissue growth, insulin resistance, respiratory,

$\triangle$ Fahrettin Akay

drfakay@yahoo.com

1 Department of Ophthalmology, İzmir Katip Çelebi University Atatürk Education and Research Hospital, İzmir, Turkey

2 Department of Ophthalmology, Balıklıgöl State Hospital, Şanlıurfa, Turkey

3 Department of Endocrinology and Metabolism, İzmir Katip Çelebi University Atatürk Education and Research Hospital, İzmir, Turkey cardiovascular, and cerebrovascular diseases [2]. In ocular tissues, growth in extraocular muscles [3], increase in intraocular pressure (IOP) [4], and increase in corneal thickness [5] have been shown previously. Also retinal thickness increase [6], increase in choroidal thickness [7], ganglion cell complex (GCC) thickness reduction [8-12], retinal nerve fibre layer (RNFL) thickness increase [6]/ decrease $[4,12]$, and increase in the incidence of proliferative retinopathy [13] have been shown previously by using optic coherence tomography (OCT) and fundus fluorescein angiography.

Endothelial dysfunction occurs in patients with acromegaly due to excess GH and IGF-1 [14]. This has been shown to cause macrovascular problems such as increased thickness of the carotid artery walls, reduced flow in the brachial artery [15], and microvascular (arteriole, capillaries, venules) problems such as increased thickness of the vessel walls [16]. IGF-1 has been accepted as a pleiotropic factor in the retina, and various effects of IGF-1 have been 
described in retinal immune modulation [17]. Optical coherence tomography angiography (OCTA) is a noninvasive imaging technique that allows to analyse and visualize retinal microvascular perfusion intensity, and to detect possible changes and to easily interpret retinal capillary network. Macrovascular and microvascular problems have been studied in specific areas such as heart and skin [14], but to our knowledge retinal microvascular changes in these patients have not been discussed before.

The aim of this study was to evaluate the retinal layers and capillary network with OCTA in acromegaly patients, to compare with healthy population, and to investigate the relationship between these structures and disease duration and serum IGF-1 levels.

\section{Materials and methods}

In this prospective, observational, and comparative study, total 40 (mean age $47.7 \pm 7.8$ years) acromegaly patients and 40 (mean age $47.3 \pm 7.2$ years) healthy control participants were included. Only right eyes of the participants were evaluated for study. All subjects were informed about the study procedure, and written consent was obtained. The study followed the tenets of the Helsinki Declaration and was approved by the institutional ethics committee.

All participants underwent a detailed ophthalmalogic examination by the same ophthalmologist (BA). All individuals with $<21 \mathrm{~mm}$ or $>24 \mathrm{~mm}$ axial length (AL), significant refractive errors ( $>3$ dioptres of spherical equivalent (SE) refraction), IOP $\geq 21 \mathrm{mmHg}$, retinal vascular diseases, uveitis, glaucoma, and pseudoexfoliation, patients who had previous ocular surgery, presence of any macular degeneration type, and smokers or ex-smokers were excluded from the analysis. For the control group, who did not have any systemic disorders, with the exception of well-controlled systemic hypertension (systolic blood pressure: $140 \mathrm{mmHg}$ and diastolic blood pressure: $90 \mathrm{mmHg}$ ). The endocrine society clinical practice guideline was used to determine the diagnosis of acromegaly [18]. Serum IGF-1 levels and disease duration of all acromegaly patients were noted.

After pupil dilatation, macular angiography was performed with $6 \times 6 \mathrm{~mm}$ area scanning using a Zeiss Cirrus 5000 system (Carl Zeiss Meditec, Dublin, CA, USA). Speed of $68,000 \mathrm{~A}$-scans per second and $840 \mathrm{~nm}$ wavelength were used for examinations. The images of the retinal capillary plexus were analysed, using Cirrus OCTA software (AngioPlex ${ }^{\mathrm{TM}}$, version 10.0) for the evaluations. The macular $6 \times 6 \mathrm{~mm}$ area was divided into sectors similar to the circles of the Early Treatment Diabetic Retinopathy Study subfields. Mean macular thickness, RNFL, and the ganglion cell inner plexiform layer (GC-IPL), vessel density (VD), perfusion density (PD), and foveal avascular zone (FAZ) parameters were measured by the same experienced operator (YZG).

Macular thickness, RNFL, and GC-IPL values obtained from scanning were calculated automatically by the device. The macular thickness was evaluated as central and mean. RNFL was evaluated by dividing into four quadrants (temporal, inferior, nasal, superior) and averaging these four quadrants. GC-IPL was divided into six regions (inferotemporal, inferonasal, superotemporal, superonasal, inferior, superior) and the average of these six regions was evaluated. For central vessel and PD, central $3 \mathrm{~mm}$ was obtained and was evaluated by dividing into three groups (inner, outer, full). FAZ parameters were evaluated dividing into three groups (area, perimeter, circularity index).

\section{Statistical analysis}

Analysis of the data was performed in the SPSS for Windows 23 (IBM Corp. Released 2012. IBM SPSS Statistics for Windows, Version 21.0. Armonk, NY: IBM Corp.) program. Before starting the study, we conducted a power analysis with the preliminary results of ten healthy and patient groups. In the preliminary results, the null hypothesis of the VD average is 22.74 (healthy group) and the alternative hypothesis of the VD average is 17.44 (patient group) and the estimated group standard deviations 6.07 and 7.29, respectively. It was observed that the sample size of 35 healthy and 35 patient groups would be sufficient by using 0.05 significance level (alpha), bilateral two-sided $t$ test to detect the difference with $90 \%$ power. The descriptive statistics were expressed as mean \pm standard deviation for variables with normal distribution, median (quartile range) for non-normal distributions, and number of cases and (\%) for nominal variables. Pearson Chi-square test and Fisher's exact test were used for comparison of descriptive statistics, as well as qualitative data. The KolmogorovSmirnov distribution test was used to examine the normal distribution. Mann-Whitney $U$ test was performed for comparison of non-normally distributed quantitative data of two groups; Student's $t$ test was used for normal distributed data. Kruskal-Wallis test was performed for comparison among more than two groups of non-normally distributed quantitative data, and Mann-Whitney U test was performed to analyse the group causing the difference. For comparison among more than two groups of normally distributed quantitative data, ANOVA test was performed and the group causing the difference is defined with post hoc Tukey test. Pearson test was used to investigate the relationship between normally distributed quantitative data; the Spearman test was used to examine the relationship between non- 
Table 1 The ocular and systemic demographics of groups.

\begin{tabular}{llll}
\hline & Control group $(n=40)$ & Acromegaly group $(n=40)$ & $p$ value \\
\hline Age, year, mean \pm SD & $47.3 \pm 7.2$ & $47.7 \pm 7.8$ & 0.502 \\
Gender male/female, $n(\%)$ & $24(60.0) / 16(40.0)$ & $16(40.0) / 24(60.0)$ & 0.117 \\
Disease duration, year, mean \pm SD & & $11.0 \pm 5.5$ & \\
IGF-1 level, ng/ml, mean \pm SD & & $208.5 \pm 84.1$ & 0.815 \\
IOP, mmHg, mean \pm SD & $16.0 \pm 1.6$ & $15.7 \pm 1.9$ & N/A \\
BCVA, logMAR mean \pm SD & $0.00 \pm 0.00$ & $0.00 \pm 0.00$ & 0.822 \\
Spherical equivalent, D, mean \pm SD & $0.31 \pm 0.65$ & $0.28 \pm 0.83$ & 0.502 \\
Axial length, mm, mean \pm SD & $22.7 \pm 0.5$ & $22.7 \pm 0.7$ & \\
\hline
\end{tabular}

$B C V A$ best corrected visual acuity, $I O P$ intraocular pressure, $I G F-1$ insulin-like growth factor-1, $D$ diopter, $\mathrm{mm}$ milimeter. normally distributed quantitative data. The results were evaluated at $95 \%$ confidence interval, $p<0.05$ significance level.

\section{Results}

Forty eyes of 40 acromegaly patients and 40 eyes of 40 healthy control subjects were evaluated. There was no significant difference between the patient group and the control group in terms of age, gender, best corrected visual acuity, $\mathrm{SE}$, IOP, and AL (Table 1). The mean follow-up period after diagnosis was $11.0 \pm 5.5$ years.

Central and mean macular thickness were also significantly higher in the acromegaly group (Table 2). Superior, inferior, and average RNFL thicknesses were also significantly thinner in the acromegaly group (Table 2). When GCC thicknesses were compared, there was no difference between the groups.

When OCTA values were compared between groups, it was observed that there was a significant decrease in central VD (CVD) and central PD (CPD) values in all regions in acromegaly group compared with control group (Table 3 ). However, when this comparison was made in FAZ values, there was no difference between the groups (Table 3 ). When the relationship between OCTA and OCT parameters was examined, there was an inverse correlation between CMT and FAZ area and perimeter values (rho: $-0.300, p$ : 0.007; rho: $-0.276, p$ : 0.013 , respectively). However, no relationship was observed between the vascularity indices (perfusion/VD) and CMT.

The correlation between disease duration and serum IGF-1 levels and parameters obtained by OCT and OCTA were investigated. There was an inverse correlation between disease duration and superior, superotemporal, inferior, inferonasal, and average GCC thicknesses (rho: -0.365 , p: 0.021 ; rho: -0.315 , p: 0.048 ; rho: -0.415 , $p$ : 0.008 ; rho: $-0.338, p$ : 0.033 ; rho: $-0.320, p$ : 0.044). No correlation was found between disease duration and parameters obtained
Table 2 Comparison of OCT parameters between groups.

\begin{tabular}{lccc}
\hline Regions & $\begin{array}{l}\text { Control group } \\
(n=40)\end{array}$ & $\begin{array}{l}\text { Acromegaly group } \\
(n=40)\end{array}$ & $p$ value \\
& \multicolumn{1}{c}{ Median (interquartile range) } & \\
\hline Mean macula & $279.5(11.0)$ & $282.5(12.0)$ & $\mathbf{0 . 0 0 8}$ \\
Central macula & $246.0(25.5)$ & $252.0(21.0)$ & $\mathbf{0 . 0 2 3}$ \\
Superior RNFL & $119.0(17.75)$ & $110.5(27.25)$ & $\mathbf{0 . 0 1 7}$ \\
Temporal RNFL & $67.0(11.5)$ & $62.0(15.0)$ & 0.194 \\
Inferior RNFL & $124.0(19.75)$ & $115.5(24.5)$ & $\mathbf{0 . 0 0 3}$ \\
Nasal RNFL & $73.5(9.75)$ & $71.0(19.0)$ & 0.350 \\
Average RNFL & $97.0(10.75)$ & $90.0(15.0)$ & $\mathbf{0 . 0 1 0}$ \\
Inferotemporal GC-IPL & $84.0(7.50)$ & $84.0(11.0)$ & 0.682 \\
Inferonasal GC-IPL & $85.0(7.0)$ & $87.0(12.75)$ & 0.350 \\
Superotemporal GC-IPL & $82.5(7.25)$ & $83.0(8.75)$ & 0.855 \\
Superonasal GC-IPL & $86.0(9.50)$ & $87.0(11.50)$ & 0.371 \\
Inferior GC-IPL & $83.0(7.75)$ & $84.0(13.50)$ & 0.357 \\
Superior GC-IPL & $85.5(9.0)$ & $84.5(12.75)$ & 0.210 \\
Average GC-IPL & $84.0(8.25)$ & $82.0(9.75)$ & 0.062 \\
Neuroretinal rim area & $1.34(0.25)$ & $1.40(0.40)$ & 0.996 \\
\hline GC-PL
\end{tabular}

$G C-I P L$ ganglion cell inner plexiform layer, $R N F L$ retinal nerve fibre layer.

Significant differences are shown in bold.

with OCTA. Central macular thickness was positively correlated with serum IGF-1 levels (rho: $0.337, p: 0.033$ ). There was no correlation between serum IGF-1 levels and OCTA values.

\section{Discussion}

Serum GH and IGF-1 levels are closely associated with complications in acromegaly patients [7, 19]. Ocular complications that can be detected and measured by OCT have been evaluated in many studies. Consistent with the results of previous studies [6,20], central macular thickness 
Table 3 Comparison of OCTA parameters between groups.

\begin{tabular}{llcr}
\hline Parameters & $\begin{array}{l}\text { Control group } \\
(n=40) \\
\text { Median (interquartile range) }\end{array}$ & $\begin{array}{l}\text { Acromegaly } \\
\text { group }(n=40)\end{array}$ & $p$ value \\
\hline Central vessel density & $11.25(4.68)$ & $9.5(1.75)$ & $\mathbf{0 . 0 4 5}$ \\
$\quad$ Inner & $19.05(0.78)$ & $18.2(2.25)$ & $<\mathbf{0 . 0 0 1}$ \\
Outer & $19.1(0.8)$ & $18.25(1.95)$ & $<\mathbf{0 . 0 0 1}$ \\
$\quad$ Full & $18.8(0.58)$ & $18.0(2.0)$ & $<\mathbf{0 . 0 0 1}$ \\
Central perfusion & $25.4(11.15)$ & $20.55(8.05)$ & $\mathbf{0 . 0 0 2}$ \\
density & & & \\
$\quad$ Inner & $45.2(2.38)$ & $43.35(4.75)$ & $<\mathbf{0 . 0 0 1}$ \\
Outer & $47.2(1.70)$ & $45.3(4.8)$ & $<\mathbf{0 . 0 0 1}$ \\
Full & $46.2(1.48)$ & $44.5(4.48)$ & $<\mathbf{0 . 0 0 1}$ \\
FAZ & & & \\
$\quad$ Area & $0.24(0.19)$ & $0.24(0.17)$ & 0.799 \\
Perimeter & $2.05(0.78)$ & $2.09(0.71)$ & 0.788 \\
Circularity index & $0.74(0.11)$ & $0.75(0.16)$ & 0.773 \\
\hline
\end{tabular}

FAZ foveal avascular zone.

Significant differences are shown in bold.

increase was also present in this study. Duru et al. stated that there was no significant difference when comparing RNFL thicknesses between healthy and acromegaly groups [12]. In contrast, Yazgan et al. reported increment in RNFL thickness in each quadrant [6]. However, Şahin et al. reported decreased RNFL thickness in inferotemporal and superotemporal quadrants [8]. In our study, there was a decrease in RNFL thickness in the inferior and superior quadrants. This difference may be due to mean disease duration differences. In addition, similar to the results in this study, when Duru et al. examined the relationship between serum IGF-1 levels and RNFL thickness, they found no correlation [12]. Şahin et al. reported an increase in GC-IPL thickness [8]. In the present study, GC-IPL thickness did not differ between the groups. This difference may be due to either the difference in disease duration or the fact that only patients without visual dysfunction were evaluated in the present study.

In experiments in mice, $\mathrm{GH}$ and IGF-I have been reported to have a direct role in vascular restructuring [21] and endothelial function [22, 23]. It has been previously mentioned that metabolic alterations characterizing acromegaly (increased prevalence of systemic arterial hypertension, alteration of glucose metabolism, and increased circulating levels of triglycerides and lipoprotein) and GH/IGF-I excess can have a direct effect on vascular structure and function [24]. In the peripheral microcirculation study performed on acromegaly patients and healthy participants, significantly more meandering capillaries with a significantly lower capillary number and length in acromegaly patients than in healthy subjects were observed [25]. Using this approach, when retinal vascular structures were evaluated in patients with acromegaly by OCTA, it was observed that there was significantly lower CVD and CPD compared with the control group. In the present study, the acromegaly group was similar to the control group in the FAZ values (area, perimeter, circularity index). In a recent study conducted with OCTA in patients with diabetes mellitus, there was a significant decrease in CVD and CPD in patients with nonproliferative and proliferative diabetic retinopathy compared with patients without diabetic retinopathy [26]. However, in the same study, FAZ values were higher in eyes with nonproliferative and proliferative diabetic retinopathy than eyes without retinopathy [26]. Other diabetic retinopathy studies have also reported decreased capillary and PD [27, 28]. The development of non-perfused capillary segments in diabetic eyes should occur for a period of time before clinical retinopathy becomes apparent [29]. Since no obvious retinopathy was seen in our acromegaly group, the similarity of the FAZ values with the control group could be explained in this way. However, a significant inverse correlation between the FAZ area and perimeter detected in this study may also be an indicator of early response to ischaemia in acromegaly patients. IGF-1, a 70-amino acid, single-chain, polypeptide macromolecule, cannot pass into the internal retina due to the tight junctions of the blood-RPE barrier. Considering the increased CMT, and the correlation between CMT and IGF1 in this study, the increase in CMT may be due to thickening in RPE. With this approach, the increase in CMT can be explained despite the decrease in the vascularity indices (perfusion/VD).

This study has some limitations, primarily the small sample size and cross-sectional design. A longitudinal study would be able to better to assess the retinal capillary network alterations in patients with acromegaly. Differences in the duration of the disease may have affected the OCTA parameters by disrupting the homogeneity of the group. Some drugs used in treatment may also have affected the current study results. Also, our findings depend on OCTA auto segmentation results. This technology has some segmentation errors. According to this, the vascularity indices (perfusion/VD) are still being established and variance may be technology platform dependent in addition to segmentation strategies.

As a result, we obtained findings consistent with the literature on OCT parameters. To the best of our knowledge, there are no studies performed with OCTA in patients with acromegaly. Our findings with OCTA show that acromegaly causes a significant capillary network decrease according to the healthy subjects. That means it causes some form of microvascular atrophy. 


\section{Summary}

\section{What was known before}

- There was no OCTA study on retinal capillary network in acromegaly patients.

\section{What this study adds}

- The present study shows that there is a significant decrease of retinal capillary density in acromegaly patients.

\section{Compliance with ethical standards}

Conflict of interest The authors declare that they have no conflict of interest.

Publisher's note Springer Nature remains neutral with regard to jurisdictional claims in published maps and institutional affiliations.

\section{References}

1. van Setten G, Brismar K, Algvere P. Elevated intraocular levels of insulin-like growth factor I in a diabetic patient with acromegaly. Orbit. 2002;21:161-7.

2. Melmed S, Colao A, Barkan A, Molitch M, Grossman AB, Kleinberg D, et al. Guidelines for acromegaly management: an update. J Clin Endocrinol Metab. 2009;94:1509-17.

3. Zafar A, Jordan DR. Enlarged extraocular muscles as the presenting feature of acromegaly. Ophthalmic Plast Reconstr Surg. 2004;20:334-6.

4. Sen E, Tutuncu Y, Elgin U, Balikoglu-Yilmaz M, Berker D, Aksakal FN, et al. Comparing acromegalic patients to healthy controls with respect to intraocular pressure, central corneal thickness, and optic disc topography findings. Indian J Ophthalmol. 2014;62:841-5.

5. Altinkaynak H, Duru N, Ersoy R, Kalkan Akcay E, Ugurlu N, Cagil N, et al. Topographic and biomechanical evaluation of cornea in patients with acromegaly. Cornea. 2015;34:65-70.

6. Yazgan S, Arpaci D, Celik HU, Isik I. Evaluation of macular and peripapillary choroidal thickness, macular volume and retinal nerve fiber layer in acromegaly patients. Int Ophthalmol. 2018;38:617-25.

7. Pekel G, Akin F, Erturk MS, Acer S, Yagci R, Hiraali MC, et al. Chorio-retinal thickness measurements in patients with acromegaly. Eye. 2014;28:1350-4.

8. Sahin M, Sahin A, Kilinc F, Yuksel H, Ozkurt ZG, Turkcu FM, et al. Retina ganglion cell/inner plexiform layer and peripapillary nerve fiber layer thickness in patients with acromegaly. Int Ophthalmol. 2017;37:591-8.

9. Ohkubo S, Higashide T, Takeda H, Murotani E, Hayashi Y, Sugiyama K. Relationship between macular ganglion cell complex parameters and visual field parameters after tumor resection in chiasmal compression. Jpn J Ophthalmol. 2012;56:68-75.

10. Monteiro ML, Hokazono K, Fernandes DB, Costa-Cunha LV, Sousa RM, Raza AS, et al. Evaluation of inner retinal layers in eyes with temporal hemianopic visual loss from chiasmal compression using optical coherence tomography. Investig Ophthalmol Vis Sci. 2014;55:3328-36.
11. Moon CH, Hwang SC, Kim BT, Ohn YH, Park TK. Visual prognostic value of optical coherence tomography and photopic negative response in chiasmal compression. Investig Ophthalmol Vis Sci. 2011;52:8527-33.

12. Duru N, Ersoy R, Altinkaynak H, Duru Z, Cagil N, Cakir B. Evaluation of retinal nerve fiber layer thickness in acromegalic patients using spectral-domain optical coherence tomography. Semin Ophthalmol. 2016;31:285-90.

13. Wu TE, Chen HS. Increased prevalence of proliferative retinopathy in patients with acromegaly. J Chin Med Assoc. 2018;81:230-5.

14. Maffei P, Dassie F, Wennberg A, Parolin M, Vettor R. The endothelium in acromegaly. Front Endocrinol. 2019;10:437.

15. De Martino MC, Auriemma RS, Brevetti G, Vitale G, Schiano V, Galdiero $\mathrm{M}$, et al. The treatment with growth hormone receptor antagonist in acromegaly: effect on vascular structure and function in patients resistant to somatostatin analogues. J Endocrinol Investig. 2010;33:663-70.

16. Lie JT. Pathology of the heart in acromegaly: anatomic findings in 27 autopsied patients. Am Heart J. 1980;100:41-52.

17. Arroba AI, Campos-Caro A, Aguilar-Diosdado M, Valverde AM. IGF-1, inflammation and retinal degeneration: a close network. Front Aging Neurosci. 2018;10:203.

18. Katznelson L, Laws ER Jr., Melmed S, Molitch ME, Murad MH, Utz A, et al. Acromegaly: an endocrine society clinical practice guideline. J Clin Endocrinol Metab. 2014;99:3933-51.

19. Guo X, Gao L, Zhang S, Li Y, Wu Y, Fang L, et al. Cardiovascular system changes and related risk factors in acromegaly patients: a case-control study. Int J Endocrinol. 2015;2015: 573643.

20. Zhang X, Ma J, Wang Y, Li L, Gao L, Guo X, et al. Elevated serum IGF-1 level enhances retinal and choroidal thickness in untreated acromegaly patients. Endocrine. 2018;59:634-42.

21. Maison P, Demolis P, Young J, Schaison G, Giudicelli JF, Chanson $P$. Vascular reactivity in acromegalic patients: preliminary evidence for regional endothelial dysfunction and increased sympathetic vasoconstriction. Clin Endocrinol. 2000;53:445-51.

22. Andersson IJ, Johansson ME, Wickman A, Bohlooly YM, Klintland N, Caidahl K, et al. Endothelial dysfunction in growth hormone transgenic mice. Clin Sci. 2006;110:217-25.

23. Bohlooly YM, Carlson L, Olsson B, Gustafsson H, Andersson IJ, Tornell $\mathrm{J}$, et al. Vascular function and blood pressure in $\mathrm{GH}$ transgenic mice. Endocrinology. 2001;142:3317-23.

24. Colao A, Ferone D, Marzullo P, Lombardi G. Systemic complications of acromegaly: epidemiology, pathogenesis, and management. Endocr Rev. 2004;25:102-52.

25. Schiavon F, Maffei P, Martini C, De Carlo E, Fais C, Todesco S, et al. Morphologic study of microcirculation in acromegaly by capillaroscopy. J Clin Endocrinol Metab. 1999;84:3151-5.

26. Rosen RB, Andrade Romo JS, Krawitz BD, Mo S, Fawzi AA, Linderman RE, et al. Earliest evidence of preclinical diabetic retinopathy revealed using optical coherence tomography angiography perfused capillary density. Am J Ophthalmol. 2019;203: 103-15.

27. Agemy SA, Scripsema NK, Shah CM, Chui T, Garcia PM, Lee JG, et al. Retinal vascular perfusion density mapping using optical coherence tomography angiography in normals and diabetic retinopathy patients. Retina. 2015;35:2353-63.

28. Spaide RF, Fujimoto JG, Waheed NK, Sadda SR, Staurenghi G. Optical coherence tomography angiography. Prog Retin Eye Res. 2018;64:1-55.

29. Krawitz BD, Mo S, Geyman LS, Agemy SA, Scripsema NK, Garcia PM, et al. Acircularity index and axis ratio of the foveal avascular zone in diabetic eyes and healthy controls measured by optical coherence tomography angiography. Vis Res. 2017;139: $177-86$. 